\title{
Influenza laboratory testing and its application in timely Department of Defense biosurveillance
}

\author{
Jessica F. Deerin, Paul E. Lewis \\ Armed Forces Health Surveillance Branch, Alexandria, Virginia, United States \\ Objective
}

To describe influenza laboratory testing and results in the Military Health System and how influenza laboratory results may be used in DoD Electronic Surveillance System for Early Notification of Community-based Epidemics (ESSENCE)

\section{Introduction}

Timely influenza data can help public health decision-makers identify influenza outbreaks and respond with preventative measures. DoD ESSENCE has the unique advantage of ingesting multiple data sources from the Military Health System (MHS), including outpatient, inpatient, and emergency department (ED) medical encounter diagnosis codes and laboratory-confirmed influenza data, to aid in influenza outbreak monitoring. The Influenza-like Illness (ILI) syndrome definition includes ICD-9 or ICD-10 codes that may increase the number of false positive alerts. Laboratory-confirmed influenza data provides an increased positive predictive value (PPV). The gold standard for influenza testing is molecular assays or viral culture. However, the tests may take 3-10 days to result. Rapid influenza diagnostic tests (RIDTs) have a lower sensitivity, but the timeliness of receiving a result improves to within $<15$ minutes. We evaluate the utility of RIDTs for routine ILI surveillance.

\section{Methods}

Administrative medical encounters for ILI and influenza laboratory-confirmed data were analyzed from the MHS from June 2013 - September 2017 (Figure 1). The medical encounters and laboratory data include outpatient, inpatient, and ED data. The ILI syndrome case definition is a medical encounter during the study period with an ICD-9 or ICD-10 codes in any diagnostic position $($ ICD-9 codes $=79.99,382.9,460,461.9,465.8,465.9,466.0,486,487.0,487.1,487.8,488,490,780.6$, or 786.2; ICD-10 codes $=$ B97.89, H66.9, J00, J01.9, J06.9, J09, J09.X, J10, J10.0, J10.1, J10.2, J10.8, J11, J11.0, J11.1, J11.2, J11.8, J12.89, J12.9, J18, J20.9, J40, R05, R50.9). The ILI dataset was limited to care provided in the MHS as laboratory data is only available for direct care. We describe influenza laboratory testing practices in the MHS. We aggregated the ILI encounters and RIDT positive results into daily counts and generated a weekly Pearson's correlation.

\section{Results}

Influenza tests are ordered throughout the year; the mean weekly percentage of ILI encounters in which an influenza laboratory test is ordered is $5.62 \%$, with a range from $0.68 \%$ in the off season to $19.2 \%$ during peak influenza activity. The mean weekly percentage of positive influenza laboratory results among all ILI encounters is $0.82 \%$, with a range from $0.01 \%$ to $5.73 \%$ (Figure 2). The percent of ILI encounters in which a test is ordered increases as the influenza season progresses. Influenza laboratory tests conducted in the MHS include RIDTs, PCR, culture, and DFA. Among all influenza tests ordered in the MHS, 66.0\% were RIDTs, $22.7 \%$ were PCR, and $11.3 \%$ were viral culture. Often, a confirmatory test is ordered following a RIDT; 20\% of RIDTs have follow-up tests. The mean timeliness of influenza test result data in the MHS was 11.26 days for viral culture, 2.94 days for PCR, and 0.11 days for RIDTs. The RIDT results were moderately correlated with ILI encounters for the entire year (mean weekly Pearson correlation coefficient rho $=0.60,95 \%$ CI: 0.55, 0.66, Figure 3). During the influenza season, the mean weekly Pearson correlation coefficient increases to rho $=0.75,95 \%$ CI: $0.70,0.79$.

\section{Conclusions}

The DoD has the unique advantage of access to the electronic health record and laboratory tests and results of all MHS beneficiaries. This analysis provides evidence for increased utilization of positive RIDTs in ESSENCE. The moderate correlation between the ILI syndrome and positive RIDTs may be associated with ICD-10 codes included in the ILI syndrome definition that contribute to false positive influenza cases. Ongoing research is focused on improving this ILI syndrome definition using ICD-10 codes. Rapid influenza diagnostic tests provide more timely results than other influenza test types. In conjunction with ILI medical encounter

SDS Annual Conference Proceedings 2019. This is an Open Access article distributed under the terms of the Creative Commons AttributionNoncommercial 4.0 Unported License (http://creativecommons.org/licenses/by-nc/3.0/), permitting all non-commercial use, distribution, and reproduction in any medium, provided the original work is properly cited. 
data, positive RIDT data provides a more complete and timely picture of the true burden of influenza on the MHS population for early warning of influenza outbreaks.

\section{Acknowledgement}

The views expressed in this article are those of the author and do not reflect the official policy or position of the Department of Defense or the U.S. Government. Support was provided by the Armed Forces Health Surveillance Branch of the Public Health Division at the Defense Health Agency.

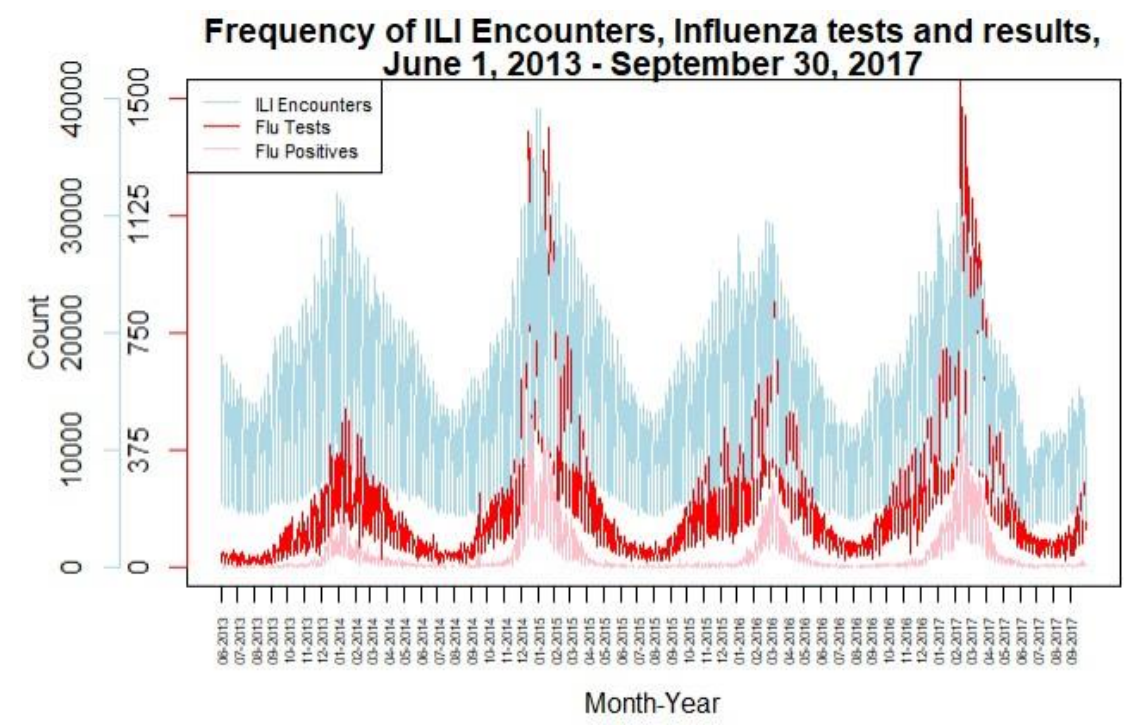

Figure 1.

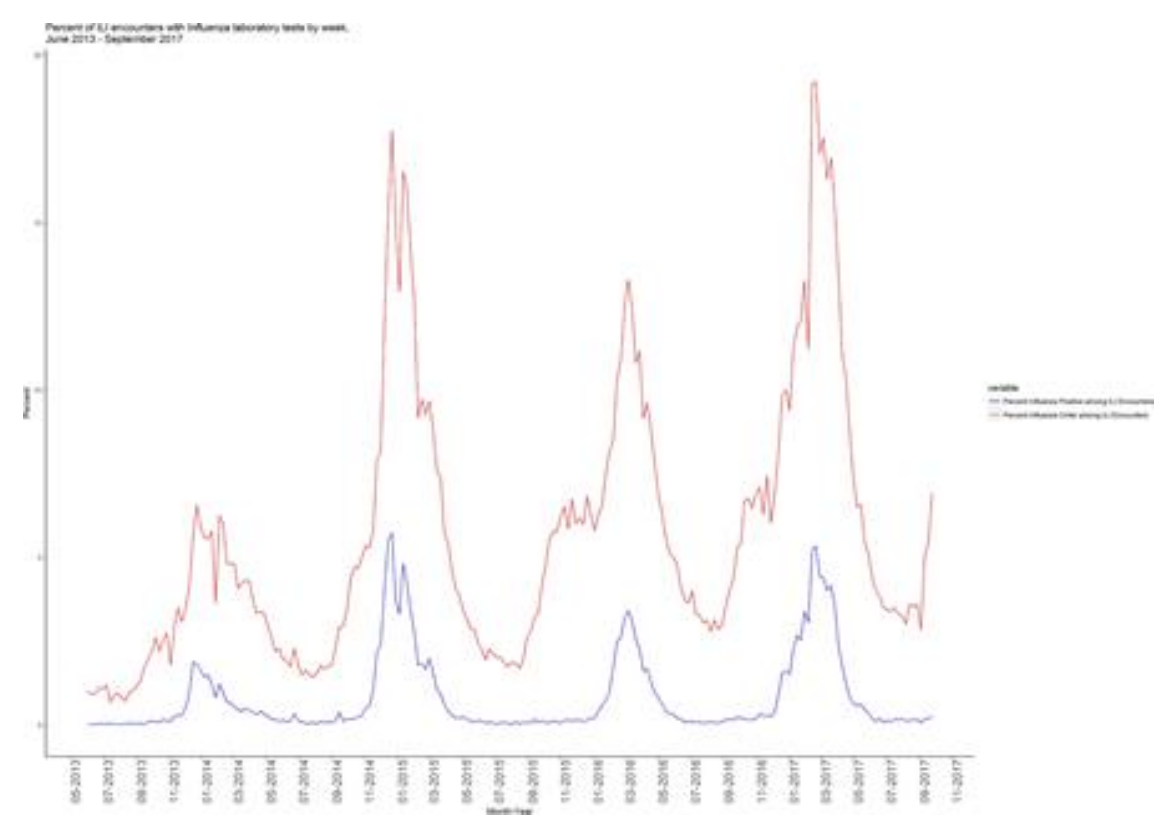

Figure 2. 


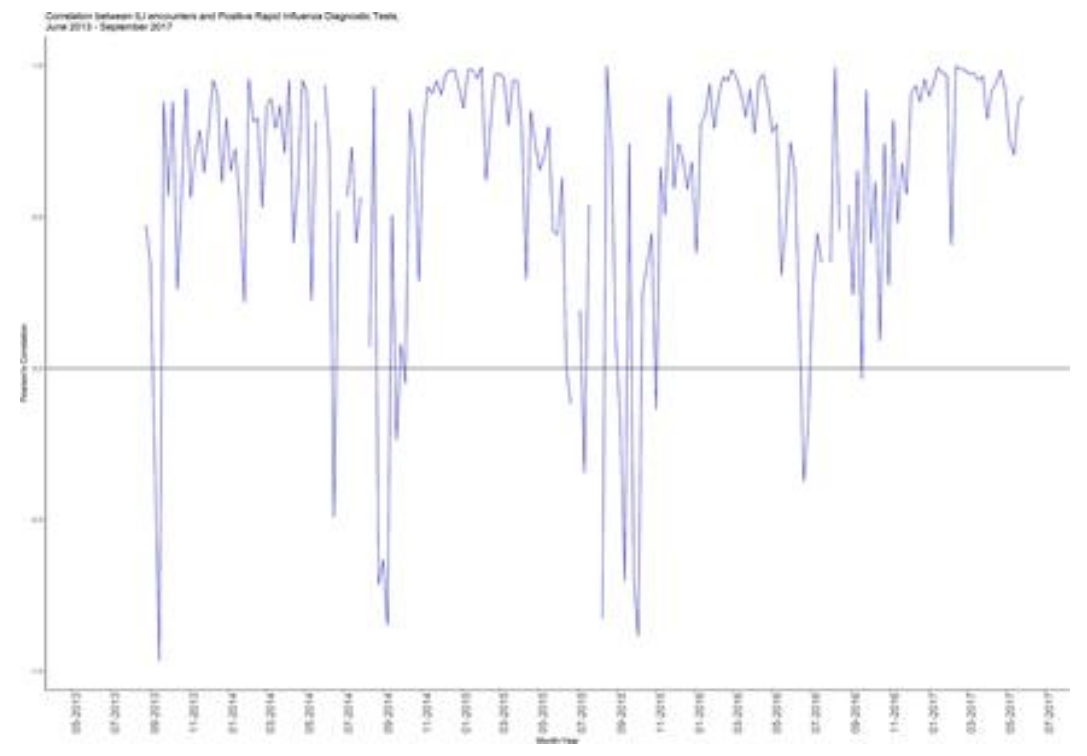

Figure 3. 